\title{
Size-Controlled Water-Soluble Ag Nanoparticles
}

\author{
José M. Domínguez-Vera, ${ }^{*[a]}$ Natividad Gálvez, ${ }^{[a]}$ Purificación Sánchez, ${ }^{[\mathrm{a}]}$ \\ Antonio J. Mota, ${ }^{[a]}$ Susana Trasobares, ${ }^{[b]}$ Juan C. Hernández, ${ }^{[b]}$ and Jose J. Calvino*[b]
}

Keywords: Silver / Nanoparticles / Apoferritin / Water-soluble particles

Ag nanoparticles of two different sizes (1 and $4 \mathrm{~nm}$ ) were prepared within an apoferritin cavity by using an $\mathrm{Ag}^{+}$-loaded apoferritin as a nanoconfined environment for their construction. The initial amount of $\mathrm{Ag}^{+}$ions injected in the apoferritin cavity dictates the size of the final Ag particles. The protein shell prevents bulk aggregation of the metal particles, which renders them water soluble and extremely stable.

(๔ Wiley-VCH Verlag GmbH \& Co. KGaA, 69451 Weinheim, Germany, 2007)

\section{Introduction}

Metallic nanoparticles are attracting considerable attention for their intriguing properties and potential applications. Because of their small size, the nanoparticles exhibit magnetic, electrical, optical, and chemical properties that cannot be achieved by bulk materials. Advances in the study of the properties of nanoparticles and in their technological applications depend on the development of new methods that are able to control the size and chemical stability of the nanoparticles. In this sense, it must be considered that traditional methods often restrict the flexibility in the preparation of small-size particles $(<10 \mathrm{~nm}){ }^{[1]}$ On the other hand, the chemical stability of the nanoparticles is crucial to avoid degradation processes such as partial oxidation or agglomeration. This is one of the reasons why noble metals have been the more usual candidates to be prepared and studied at the nanometer regimen. Among noble metals, $\mathrm{Au}$ and Ag exhibit a strong UV/Vis absorption band that is not present in the spectrum of the bulk metals. This absorption band results when the incident photon frequency is resonant with the collective excitation of the conduction electrons and is known as the surface plasmon resonance (SPR). ${ }^{[2]}$ The SPR can be tuned by changing particle size, shape, as well as the surrounding dielectric medium. This tunability confers to $\mathrm{Au}$ and $\mathrm{Ag}$ nanoparticles potential applications for surface-enhanced spectroscopy, ${ }^{[3]}$ optical filters, ${ }^{[4]}$ and plasmonic devices ${ }^{[5]}$ and sensors. ${ }^{[6]}$ This potentiality is exploited more in the case of $\mathrm{Au}$ nanoparticles than it is for $\mathrm{Ag}$ nanoparticles despite the known fact that

[a] Departamento de Química Inorgánica, Facultad de Ciencias, Universidad de Granada,

Fuentenueva s/n 18071 Granada, Spain

Fax: +34-958-248526

E-mail: josema@ugr.es

[b] Departamento Ciencia de Materiales e Ingeniería Metalúrgica y Química Inorgánica, Universidad de Cádiz,

Campus Río San Pedro, 11510 Cádiz, Spain

E-mail: jose.calvino@uca.es
Ag nanoparticles exhibit higher efficiency of plasmon excitation. ${ }^{[2]}$ However, the propensity of pure Ag nanoparticles to undergo oxidative corrosion ${ }^{[7]}$ and aggregation in electrolytic solutions ${ }^{[8]}$ limit their usage, especially for biological applications. The oxidative corrosion and aggregation of $\mathrm{Ag}$ nanoparticles can be attenuated or eliminated with a protective layer, such as organic cap ligands or inorganic coatings, which allow stable nanoparticle solutions to exist even at high $\mathrm{NaCl}$ concentrations and over a wide range of $\mathrm{pH}$ values. An alternative route for avoiding the agglomeration of the particles is the use of a preorganized molecular matrixas a chemical and spatial nanocage for building the Ag nanoparticle. An example of this underexploited route is the use of genetically engineered apoferritin expressing a silverbinding peptide. ${ }^{[9]}$ Native apoferritin consists of a spherical protein shell surrounding an aqueous cavity of about $8 \mathrm{~nm} \cdot{ }^{[10]}$ It was reported that apoferritin binds or concentrates metal ions in its cavity ${ }^{[11]}$ and that, interestingly, the loaded metal ions can be chemically reduced to form the corresponding zero-valent cluster, which remains encapsulated. ${ }^{[12]}$ Moreover other inorganic nanoparticles (especially oxides and hydroxides) can be prepared within the apoferritin cavity by using different strategies. ${ }^{[13]}$ The final particle size should be directly determined by the number of metal ions previously encapsulated. However, size tuning by metal ion loading control was only reported for native ferritin, which was prepared with a different iron content. ${ }^{[14]} \mathrm{We}$ herein report the preparation of apoferritin-encapsulated silver nanoparticles, and we demonstrate that the initial amount of $\mathrm{Ag}^{+}$ions injected in the apoferritin capsule dictates the size of the resulting Ag particles.

\section{Results and Discussion}

Sodium chloride free horse spleen apoferritin was incubated with $\mathrm{AgNO}_{3}$ at two different stoichiometries (500 and 
$5000 \mathrm{Ag}^{+}$per apoferritin) to afford homogeneous solutions that were chromatographed (Sephadex G-25). Every collected fraction was added to a $\mathrm{NaBH}_{4}$ solution and the apoferritin and $\mathrm{Ag}$ concentrations were then measured (see Experimental Section). The elution profile does not change with the $\mathrm{Ag}^{+}$/apoferritin stoichiometry. As an example, the elution profile of the solution corresponding to the experiment of higher $\mathrm{Ag}^{+}$/apoferritin is shown in Figure 1. The Ag-associated apoferritin peak (fractions 4-7) was separated from free $\mathrm{Ag}$ (fractions 10-12), which trailed well behind (Figure 1). The coelution was an indication that $\mathrm{Ag}$ was attached to apoferritin. In fact, fraction 4-7 contained water-soluble particles of sizes smaller than the apoferritin cavity (see below) whereas fractions 10-12 consisted of insoluble and heterogeneous larger Ag particles (20-80 nm).
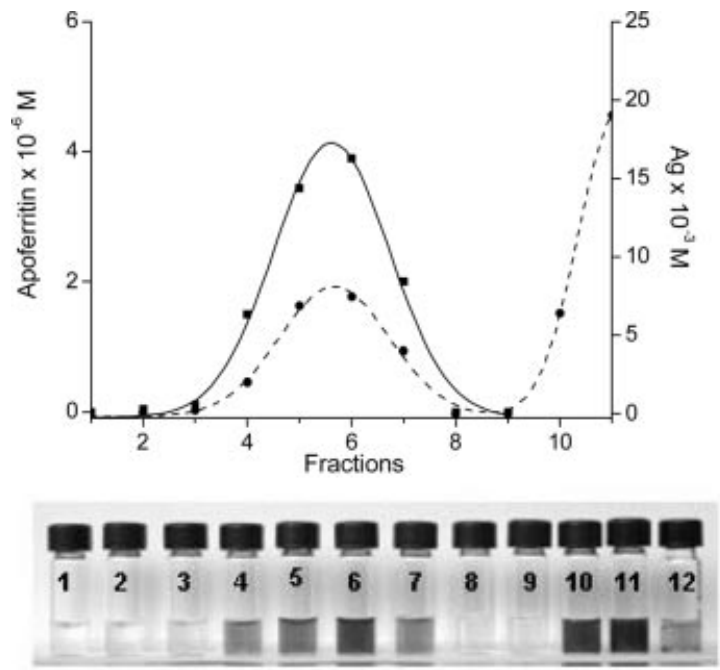

Figure 1. Elution profile of Ag-containing apoferritin. Protein (solid line) was measured by UV/Vis spectroscopy and Ag (dashed line) by atomic absorption spectroscopy.

The solutions corresponding to fractions 5-7 were dialyzed against water at $4{ }^{\circ} \mathrm{C}$ and then examined by transmission electron microscopy (TEM). A typical image obtained from the material corresponding to the experiments made in a $5000: 1 \mathrm{Ag}^{+} /$apoferritin stoichiometry shows discrete electron-dense cores, which are spherical in shape (Figure 2). The mean diameter was statistically measured to be $(4 \pm 0.5) \mathrm{nm}$. X-ray energy dispersive spectroscopy (EDS) confirmed that the particles contained Ag (Figure 2d), which was not detected outside the particles. TEM images of samples negatively stained with uranyl acetate (to visualize the protein shell) confirmed that the particles were actually produced within the apoferritin interior (Figure $2 b$ ). The presence of the apoferritin coat prevents irreversible aggregation of the $\mathrm{Ag}$ particles and their precipitation. For this reason, apoferritin-free Ag particles (fractions 10 and 11) resulted in bulk precipitation.

The use of the apoferritin cavity as a nanoreactor to build the Ag particle allows initial control over the size, because the particles cannot be larger than the cavity (ap-
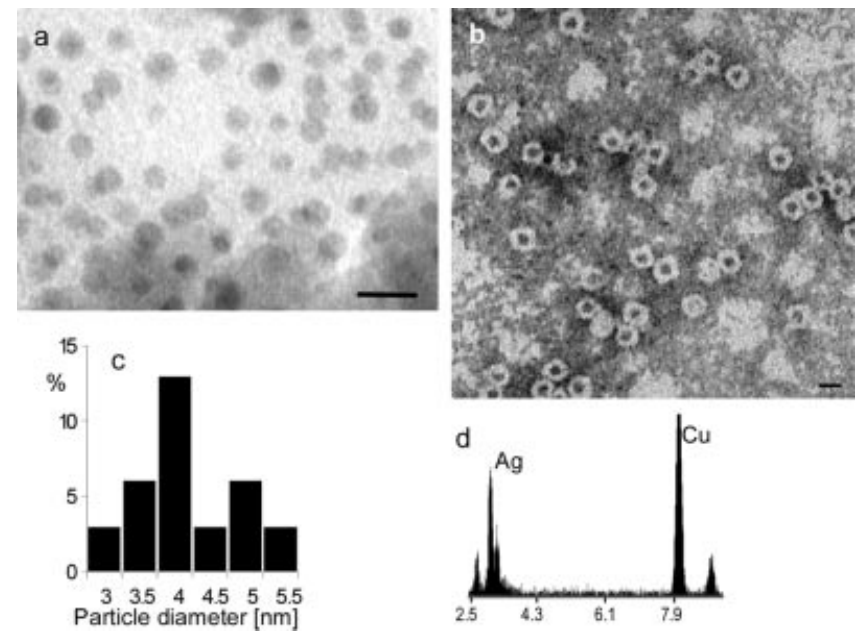

Figure 2. (a) TEM image of $\mathrm{Ag}$ nanoparticles prepared in a $\mathrm{Ag} /$ apoferritin stoichiometry of 5000:1; (b) negatively stained TEM image showing the metallic core surrounded by the apoferritin shell (scale bars are $10 \mathrm{~nm}$ ); (c) size-distribution in $\mathrm{nm}$; and (d) representative EDS recorded inside the particles. $\mathrm{Cu}$ peaks are due to the sample grid.

proximately $8 \mathrm{~nm}$ ). In fact, particles from fractions $5-7$ are smaller than $8 \mathrm{~nm}$, whereas those of the apoferritin-free ones (fractions 10-12) are significantly larger (20-80 nm). However, more exhaustive control over the particle size is possible: the initial amount of $\mathrm{Ag}^{+}$injected in the apoferritin cavity plays a key role in dictating the size of the particles and then the size may be tuned by changing the molar ratio of $\mathrm{Ag}^{+}$/apoferritin. Thus, with a lower $\mathrm{Ag}^{+}$/apoferritin ratio $(500: 1)$ and the use of the same experimental procedure (incubation time, chromatography, and dialysis), particles of smaller size were obtained. The detection and structural characterization of these smaller particles requires the use of alternative electron microscopy techniques. Given the large difference in atomic number between $\mathrm{Ag}(Z$ $=47)$ and $C(Z=6)$, high angle annular dark field imaging (HAADF) was used, which extends the detection limit below the nanometer range. Figure 3 a shows a representative HAADF image of the $\mathrm{Ag}^{+} /$apoferritin (500:1) sample. Bright spot contrasts correspond to the high atomic number component, namely, the silver particles. As shown in Figure 3a, a large number of nanosized Ag particles can be detected in the HAADF images.

EDS obtained from locations inside these particles by using a finely focused $0.5-\mathrm{nm}$ electron probe (Figure $3 \mathrm{~b}$ ) confirm the presence of $\mathrm{Ag}$ in these bright areas. From the particle size histogram established from HAADF images (Figure 3c) an average Ag particle size of $(1 \pm 0.1) \mathrm{nm}$ can be estimated in this sample.

The structure of the small particles containing Ag was further investigated by means of high-resolution electron microscopy (HREM). Figure 4 shows some representative HREM images. Measurements of lattice spacings and interplanar angles on the diffractograms of these images (included as insets) confirm that the particles are fcc Ag. Some 

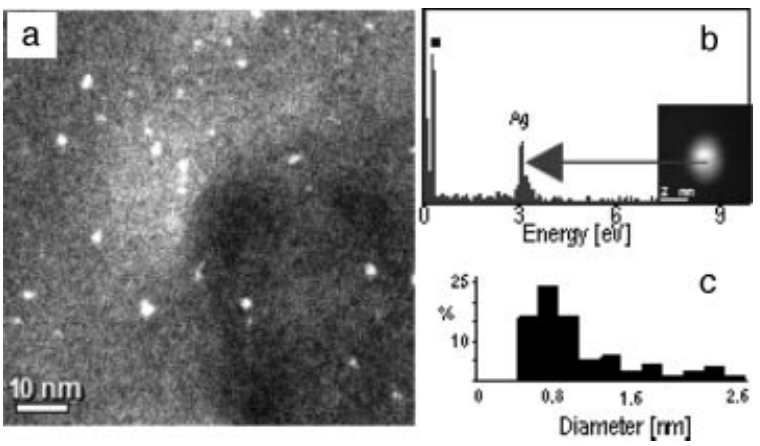

Figure 3. (a) STEM-HAADF image of the 500:1 $\mathrm{Ag}^{+} /$apoferritin sample; (b) X-EDS spectrum obtained from inside one of the particles (shown as inset); (c) particle diameter histogram of this sample established from a collection of HAADF images.

of them are monocrystalline domains (Figure 4a,b), whereas in some other cases they are made of several singly or multiply twinned smaller fcc domains (Figure 4c,d). In the diffractograms of the former, $\{002\}$ reflections at $0.204 \mathrm{~nm},\{022\}$ at $0.14 \mathrm{~nm}$, and $\{111\}$ at $0.235 \mathrm{~nm}$ can be clearly identified. Twinning on the $\{111\}$ planes complicates the appearance of the diffractograms in the case of the latter, but these same reflections can still be clearly identified. Note that the size of the domains in these twinned particles is smaller than $1 \mathrm{~nm}$.

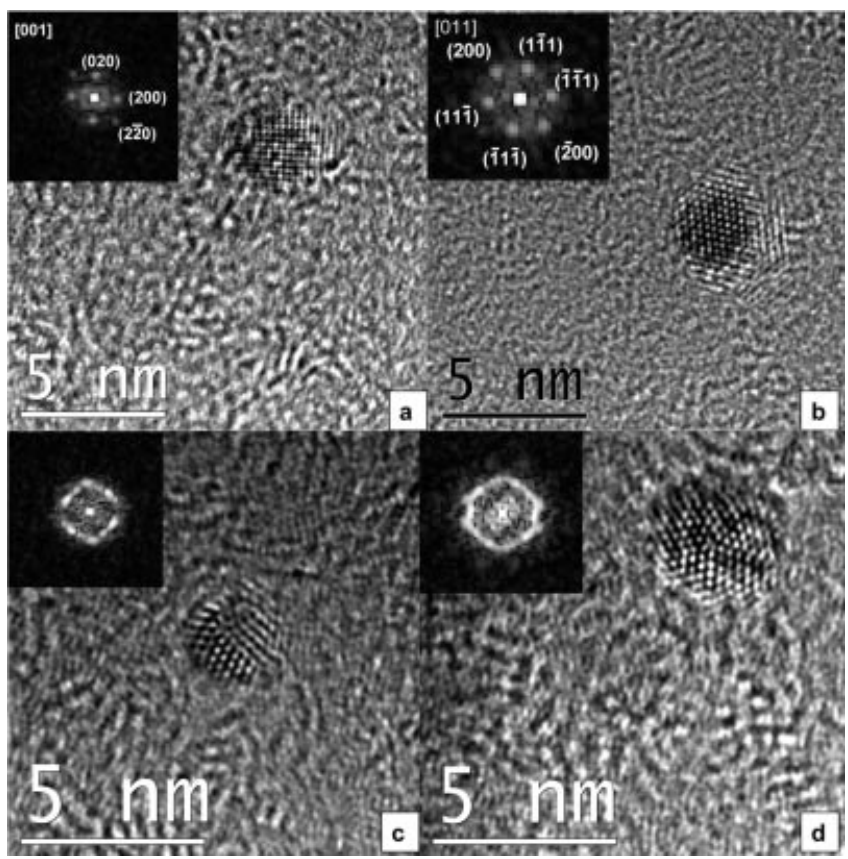

Figure 4. HREM images recorded of the 500:1 $\mathrm{Ag}^{+}$/apoferritin sample: (a) shows a fcc Ag particle down the [001] zone axis; (b) Ag particle down the [110] zone axis; (c) and (d) correspond to multiply twinned Ag particles. Diffractograms are included as insets. Multiply-twinned particles illustrate the structure of larger particles.
These results demonstrate that $\mathrm{Ag}^{+}$-apoferritin acts as a reactor because the trapped $\mathrm{Ag}^{+}$ions are able to react with a chemical reagent that is small enough to traverse the apoferritin channels to build a new material. Thus, $\mathrm{Ag}^{+}$ions are reduced with $\mathrm{NaBH}_{4}$ to yield zero-valent silver. As a result of the reduction, the UV/Vis spectrum of the solution dramatically changed and showed the typical absorption band attributable to the SPR of the Ag particles. The position of the SPR can be tuned by changing the particle size and shape, as well as the surrounding dielectric medium. In Figure 5, the UV/Vis spectra of the solution corresponding to the particles of 1 and $4 \mathrm{~nm}$ show the presence, in both cases, of the SPR at 392 and $434 \mathrm{~nm}$, respectively. This significant shift must be a direct consequence of the different particle sizes ${ }^{[15]}$ and/or their different dielectric surrounding media: the lower the Ag particle size, the less contact with the apoferritin takes place. In fact, particles of $1 \mathrm{~nm}$ exhibit a SPR $(392 \mathrm{~nm})$ that is typical for uncoated Ag nanoparticles, whereas that $(434 \mathrm{~nm})$ of the $4-\mathrm{nm}$ sized particle is more characteristic of $\mathrm{Ag}$ particles surrounded with a high refractive index shell. ${ }^{[16]}$

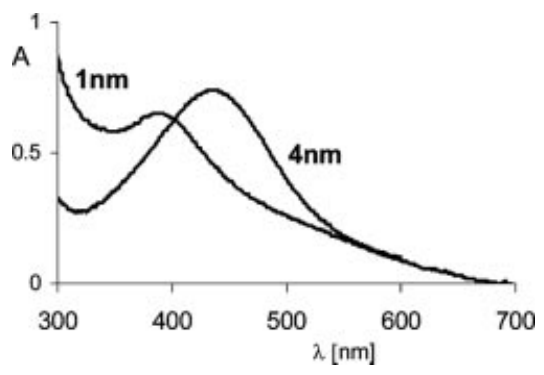

Figure 5. UV/Vis spectra of the Ag particles. The SPR showed a redshift with a reduced particle size.

Stability is a key point for silver nanoparticles. Previous studies showed that silver nanoparticles were often unstable in solution and easily aggregated at high concentration of the particles or $\mathrm{NaCl}$. The apoferritin-encapsulated $\mathrm{Ag}$ nanoparticles we have prepared exhibited excellent stability in solution and no significant UV/Vis spectral changes were observed over one month at room temperature. Likewise, the addition of $\mathrm{NaCl}$ to reach a $0.1 \mathrm{M}$ concentration did not produce either precipitation or UV/Vis spectral change. Moreover, the solution can be lyophilized and the particles kept in the solid state under ambient conditions for months without showing significant changes on the basis of X-ray powder diffraction analysis.

\section{Conclusions}

Ag nanoparticles were prepared by using the apoferritin cavity as a nanoreactor to reduce the $\mathrm{Ag}^{+}$ions. The size of the nanoparticles can be tuned by changing the $\mathrm{Ag}^{+}$loading. The presence of the apoferritin coat is responsible for the water solubility and stability of these particles. 


\section{Experimental Section}

Horse spleen apoferritin $\left(53 \mathrm{mg} \mathrm{mL}^{-1}\right)$ was exhaustively dialyzed for $3 \mathrm{~d}$ with several changes of the milli-Q water at $4{ }^{\circ} \mathrm{C}$ to remove $\mathrm{NaCl}$. After this treatment, apoferritin $\left(5.3 \mathrm{mg} \mathrm{mL}^{-1}\right)$ was incubated with $\mathrm{AgNO}_{3}$ at two different concentrations (6 and $60 \mathrm{~mm}$ ) at room temperature, which corresponds to 500 and $5000 \mathrm{Ag}^{+} /$apoferritin stoichiometries. No precipitation of $\mathrm{AgCl}$ occurred. After $1 \mathrm{~d}$, the resulting solutions were dialyzed against water for $15 \mathrm{~min}$ and then passed through a $1.5 \mathrm{~cm} \times 5.5 \mathrm{~cm}$ column of Sephadex G25 equilibrated with water. Fractions were collected every minute, and then, the concentrations of apoferritin and $\mathrm{Ag}$ were obtained by the absorbance values at $280 \mathrm{~nm}\left(\varepsilon^{280} \approx 468000 \mathrm{M}^{-1}\right)$ and atomic absorption spectroscopy, respectively. In a parallel experiment, the chromatographed fractions were directly added to a water solution containing $\mathrm{NaBH}_{4}\left(4.5 \mathrm{mg} \mathrm{mL}{ }^{-1}\right)$. The samples used for electron microscopy (TEM) studies were prepared by diluting the dialyzed solutions of particles with milli-Q water and then placing a drop onto a carbon-coated $\mathrm{Cu}$ grid and drying it in a glove box. To record images in high-resolution electron microscopy (HREM) and high angle annular dark field (HAADF) modes, the samples were deposited onto holey carbon $\mathrm{Cu}$ grids. The average particle sizes and the standard deviations were estimated from measurements of diameters performed either with TEM or HAADF images of a statistically adequate number of particles (about a hundred). Electron micrographs were recorded with two different microscopes: a Philips CM-20 HR analytical electron microscope operating at $200 \mathrm{keV}$ (TEM) and a JEOL 2010F (HREM, HAADF) working also at $200 \mathrm{kV}$. Structural resolution of the last instrument was $0.19 \mathrm{~nm}$, which allowed structural analysis of noble metals as it is the case of silver. Moreover operating this instrument in scanning transmission electron microscopy mode (STEM) allows forming electron probes with diameters as small as $0.2 \mathrm{~nm}$, which opens the possibility, in combination with X-EDS detectors, to perform chemical analysis with a spatial resolution better than $1 \mathrm{~nm}$.

\section{Acknowledgments}

Financial support from the Spanish Ministerio de Educación y Ciencia (project CTQ2006-02840) and Junta de Andalucía (Proyecto Excelencia FQM-00425 and FQM-334) are gratefully acknowledged. N. G. and S. T. thank the Spanish Ministerio de Ciencia y Tecnología for a research contract (programa Ramón y Cajal).

[1] D. D. Evanoff Jr, G. Chumanov, ChemPhysChem 2005, 6, 1221-1231; I. Sondi, D. V. Goia, E. Matijevic, J. Colloid Interface Sci. 2003, 260, 75-81; M. P. Pileni, J. Phys. Chem. 1993, 97, 6961; N. Shirtcliffe, U. Nickel, S. Schneider, J. Colloid Interface Sci. 1999, 211, 122-129; K. P. Velikov, G. E. Zegeres, A. V.
Blaaderen, Langmuir 2003, 19, 1384-1389; Y. Tan, X. Dai, Y. Li, D. Zhu, J. Mater. Chem. 2003, 13, 1069-1075; K. K. Caswell, C. M. Bender, C. J. Murphy, Nano Lett. 2003, 3, 667669; D. L. Van Hyning, C. F. Zukoski, Langmuir 1998, 14, 7034-7046.

[2] U. Kreibig, M. Vollmer in Springer Series in Materials Science Vol. 25: Optical Properties of Metal Clusters, New York, Springer, 1995.

[3] G. C. Schatz, R. P. Van Duyne in Electromagnetic Mechanism of Surface-Enhanced Spectroscopy, 2002, Wiley, New York, vol. 1; C. L. Haynes, R. P. Van Duyne, J. Phys. Chem. B 2003, 107, 7426-7433; Z.-G. Shang, D. N. Ting, Y. T. Wong, Y. C. Tan, B. Ying, Y.-J. Mo, J. Mol. Struct. 2007, 826, 64-67.

[4] Y. Dirix, C. Bastiaansen, W. Caseri, P. Smith, Adv. Mater. 1999, 11, 223-227; C. L. Haynes, R. P. Van Duyne, Nano Lett. 2003, 3, 939-943.

[5] S. A. Maier, M. L. Brongersma, P. G. Kik, S. Meltzer, A. A. G. Requicha, H. A. Atwater, Adv. Mater. 2001, 13, 1501-1505; R. A. Shelby, D. R. Smith, S. Schultz, Science 2001, 292, 7778.

[6] A. J. Haes, R. P. Van Duyne, J. Am. Chem. Soc. 2002, 124, 10596-10604; R. C. Mucic, J. J. Storhoff, C. A. Mirkin, R. L. Letsinger, J. Am. Chem. Soc. 1998, 120, 12674-12675.

[7] A. Henglein, Chem. Mater. 1998, 10, 444.

[8] T. Huang, R. W. Murray, J. Phys. Chem. B 2003, 107, 7434.

[9] R. M. Kramer, C. Li, D. C. Carter, M. O. Stone, R. R. Naik, J. Am. Chem. Soc. 2004, 126, 13282-13286.

[10] P. M. Harrison, P. Arosio, Biochim. Biophys. Acta 1996, 1275, 161; P. M. Proulx-Curry, N. D. Chasteen, Coord. Chem. Rev. $1995,144,347$.

[11] S. Pead, E. Durrant, B. Webb, C. Larsen, D. Heaton, J. Johnson, G. D. Watt, J. Inorg. Biochem. 1995, 59, 15.

[12] N. Gálvez, P. Sánchez, J. M. Domínguez-Vera, Dalton Trans. 2005, 2492; N. Gálvez, P. Sánchez, J. M. Domínguez-Vera, A. Soriano-Portillo, M. Clemente-León, E. Coronado, J. Mater. Chem. 2006, 16, 2757; M. Clemente-Leon, E. Coronado, A. Soriano-Portillo, N. Galvez, J. M. Dominguez-Vera, J. Mater. Chem. 2007, 17, 49-51.

[13] K. Yoshizawa, K. Iwahori, K. Sugimoto, I. Yamashita, Chem. Lett. 2006, 35, 1192; K. Iwahori, T. Morioka, I. Yamashita, Phys. Status Solid A 2006, 203, 2658; K. Iwahori, K. Yoshizawa, M. Muraoka, I. Yamashita, Inorg. Chem. 2005, 44, 6393; M. Uchida, M. T. Klem, M. Allen, P. S. uci, M. Flenniken, E. Gillitzer, Z. Varpness, L. O. Liepold, M. Young, T. Douglas, Adv. Mater. 2007, 19, 1025 and references cited therein.

[14] S. Gider, D. D. Awschalon, T. Douglas, S. Mann, M. Chaparala, Science 1995, 268, 77.

[15] A. S. Kumbhar, M. K. Kinnan, G. Chumanov, J. Am. Chem. Soc. 2005, 127, 12444-12445; S. Hussain, R. K. Roy, A. K. Pal, Mater. Chem. Phys. 2006, 99, 375.

[16] I. Pastoriza-Santos, D. S. Koktysh, A. A. Mamedov, M. Giersig, N. A. Kotov, L. M. Liz-Marzán, Langmuir 2000, 16, 27312735 .

Received: June 11, 2007

Published Online: August 28, 2007 